\title{
Experimentally induced errors in Digital Image Correlation measurement of small strains with large gradients
}

\author{
I. De Baere ${ }^{1}$, W. Van Paepegem ${ }^{1}$ N. Lammens ${ }^{1}$ P. Lava ${ }^{2}$, D. Debruyne ${ }^{2}$, C. Cofaru ${ }^{1,3}$, W. \\ Philips $^{3} \&$ J. Degrieck ${ }^{1}$
}

${ }^{1}$ Ghent University, Department of Materials Science and Engineering, Technologiepark-Zwijnaarde 903, 9052 Zwijnaarde, Belgium

${ }^{2}$ Catholic University College Ghent,Department of Mechanical Engineering, Gebroeders De Smetstraat 1, 9000 Ghent, Belgium

${ }^{3}$ Ghent University, Department of Telecommunications and Information Processing. Sint-Pieternieuwstraat 41, 9000 Ghent, Belgium

ABSTRACT: Digital Image Correlation or DIC is an already widespread and commonly used technique to perform full field strain measurements. Usually, a so called 'speckle pattern' is put on the specimen and then, pictures are taken first in the undeformed stage, which is the reference and then in various deformed states. The technique itself consists of comparing the image of a deformed pattern with a reference image and determining the displacements of the so called 'subsets'. The mathematical algorithm eliminates rigid rotations of the subset and is able to determine the strain field. This technique was originally designed for large-strain measurements and as such, it works very well when large strains are present, but when determining (very) small strain fields, especially in combination with large (rigid body) deformations or large strain gradients, this technique becomes a lot more sensitive to the boundary conditions of the experimental setup.

This manuscript will illustrate the errors induced by experimental factors such as in-plane rotation, in-plane rigid body translation, out-of-plane rigid body rotation, which are in fact all related to how the specimen is gripped in the tensile machine, on the eventual derived strain field. Furthermore, processing parameters such as subset and step size and the used strain window on numerically and experimentally induced transformations will be assessed.

\section{INTRODUCTIONS}

Digital image correlation (DIC) offers unique opportunities for exploring full-field displacements and strain measurements and this for a wide range of materials (Lava et al. 2009, Van Paepegem et al. 2009, Willems et al. 2009).

In most cases, DIC is used to determine very large strains, e.g. large plastic strains in metals. In this case, the strains are so large that they cause displacement in the order of pixels. For composites, however, strains are a lot smaller. Usually, failure strains are around 1 to $1.5 \%$ and the corresponding displacement is quite often in the sub-pixel range. The question is whether the DIC technique is still accurate enough to determine the occurring strain levels. Furthermore, because of the inhomogeneous nature of composites, the strain will not be uniform, but will have gradients, especially for fabric reinforcements. Indeed, the stiffer fibres will carry more load, but still deform less than the weaker matrix. The goal of current research project would be to use DIC to determine the strain fields of an undamaged composite and then compare it with the strain field of the same specimen, in which some form of (fatigue) damage has accumulated. However, before this is attempted, it must first be determined whether DIC is accurate enough and what the influence is of experimental and/or numerical factors such as rigid body transformations and gripping of the specimen. Therefore, this manuscript considers a number of 'predictable tests' in order to assess the DIC technique.

\section{MATERIALS AND METHODS}

For the tensile specimens, a 5-harness satin-weave carbon fabric-reinforced polyphenylene sulphide (PPS) was considered. The carbon PPS plates were hot pressed, and one stacking sequence was used for this study, namely $\left[\left(0^{\circ}, 90^{\circ}\right)\right]_{2 s}$ where $\left(0^{\circ}, 90^{\circ}\right)$ represents one layer of fabric; Figure 1 illustrates the woven structure. This material was supplied to us by Ten Cate Advanced Composites (The Netherlands).

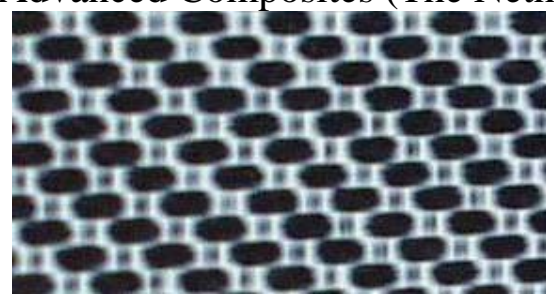

Figure 1 illustration of the CETEX 5-harness satin weave 
All images were taken with a PixeLink digital camera with a 6.6Megapixel CCD chip and the Digital image correlation was done using the MatchID software (Lava et al. 2009).

\section{EXPERIMENTS AND DISCUSSION}

\subsection{DIC on CETEX tensile test}

A tensile test was performed on the CETEX material on an electromechanical Instron and it was attempted to derive the strain fields using DIC. Figure 2 illustrates the displacement and strain fields for a stress level of $790 \mathrm{MPa}\left(\varepsilon_{\mathrm{xx}}=1.4 \%\right)$, which is just before failure; the black lines on top of the image are the rubber bands of the extensometer which was mounted. Two remarks can be made: first, the displacement fields show unexpected diagonal bands, of which the origin lies most likely in the used tensile machine. Indeed, for the used electromechanical Instron, the grips are allowed to self align during the test. Since this is a rigid body transformation, it should not have an influence on the derived strain fields. Second, the expected weave pattern of the 5harness satin weave (Fig. 1) is clearly visible, although the strain levels vary from top to bottom, which is not accurate. Changing subset and step sizes and the strain window do not change this effect, so most likely, it is induced by either the test or the software.

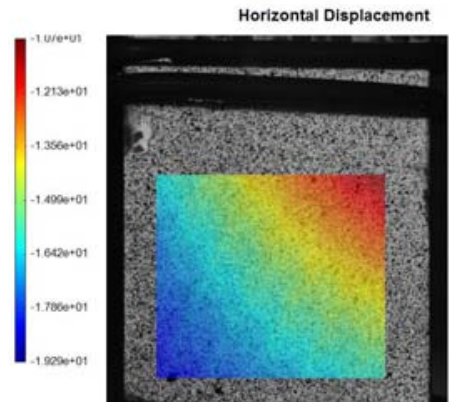

(a) horizontal displacement

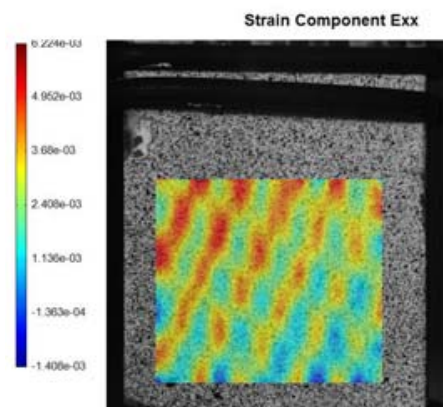

(c) horizontal strain $\varepsilon_{\mathrm{xx}}$

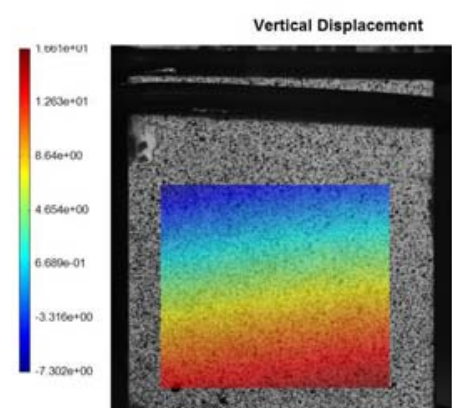

(b) vertical displacement

Strain Component Eyy

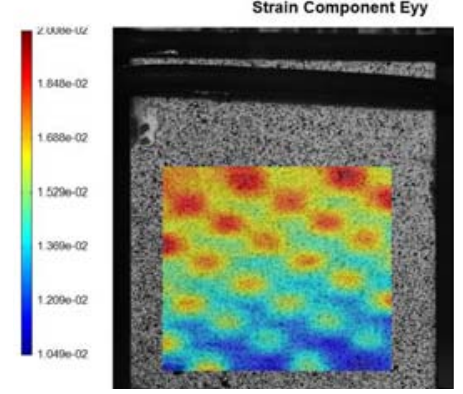

(d) vertical strain $\varepsilon_{\mathrm{yy}}$
Figure 2 DIC on a tensile test on the CETEX material (electromechanical Instron)
To check the influence of the tensile machine, a similar experiment was done on a servo-hydraulic Instron with fixed grips and an anti-rotation fixture; the grips were aligned using a special Instron alignment kit. Although this improves the quality of the displacement and strain fields (Fig. 3), the phenomenon is still present and the reasons are unclear, since no rigid body transformations should occur.

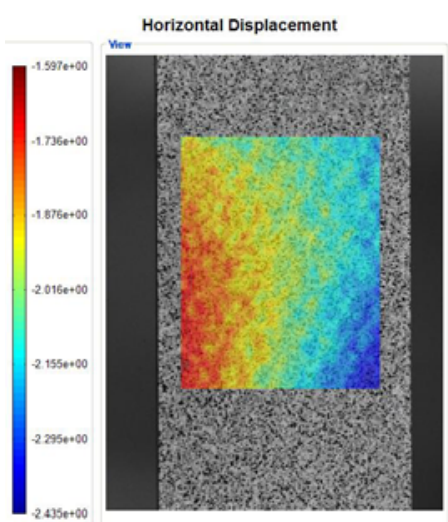

(a) horizontal displacement

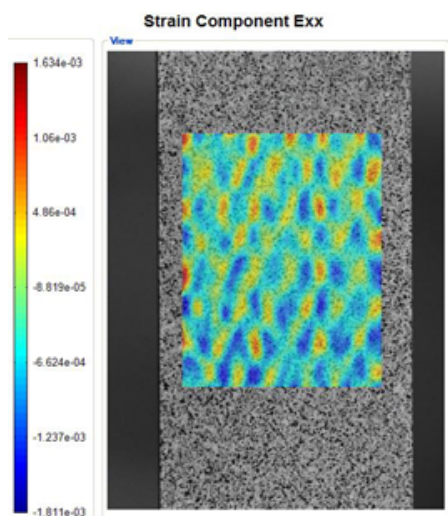

(c) horizontal strain $\varepsilon_{\mathrm{xx}}$

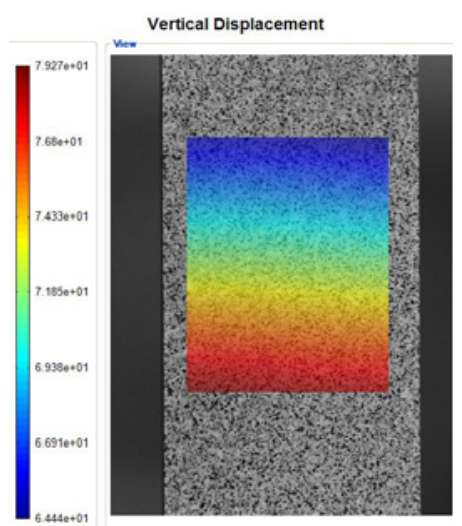

(b) vertical displacement

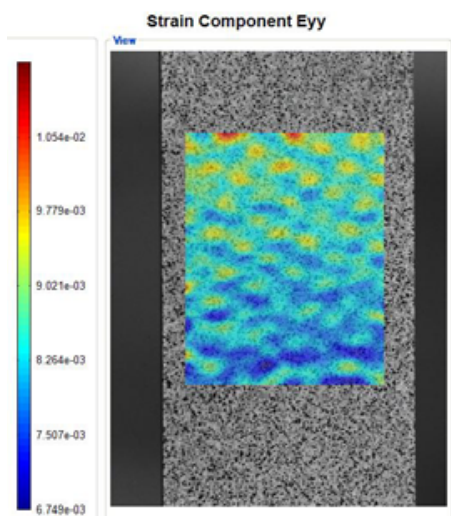

(d) vertical strain $\varepsilon_{y y}$
Figure 3 DIC on a tensile test on the CETEX material (hydraulic Instron)

Since the purpose is to use this technique to accurately determine strain fields prior and during a fatigue experiment in order to assess the occurring damage by small changes in the strain fields, the accuracy must be as high as possible and the parameters which induce the above indicated errors must be determined and if possible, eliminated.

\subsection{DIC on experimentally induced transformations}

Since a number of strange and inexplicable effects were visible in the DIC measurements on a tensile test, it was decided to take one step back and to apply simple rigid body transformations, such as pure translation and pure rotation inside the tensile machine. Theoretically, the correlation algorithm is supposed to eliminate such rigid body deformations

With respect to the speckle pattern and size, Figure 4(a) illustrates a detailed image of the speckle size. As can be noted, all speckles consisted of a lar- 
ger number of pixels, ensuring good correlation. Figure 4(b) shows a general overview of the correlation area, as well as the chosen subset, which has a size of 55 pixels. This value, combined with a step size of 17 proved to give valid results and concerning the quality of the speckle, for all mentioned transformations, the correlation factor varied between 0.99 and 1 , meaning very good correlation is achieved.

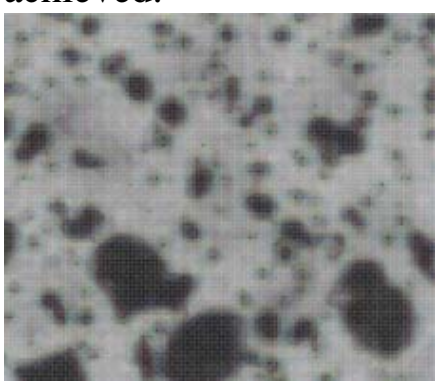

(a) speckle size

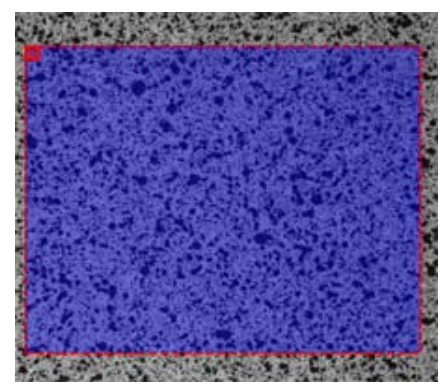

(b) subset size
Figure 4 Illustration of speckle pattern and chosen subset size

For a first test a vertical rigid body translation of $1 \mathrm{~mm}$ was imposed. The results are illustrated in Figure 5, the illustrated strains were calculated with a strain window of 5 (noted $w=5$ ). Although i) the vertical displacement is fairly constant and about 41 pixels and ii) the horizontal displacement is very small (about 0.2 pixels) and constant, a fairly large parasitic strain is present. The average value of both strains is in the order of $110^{-5}$, which is of course, acceptable, but the amplitude is in the order of 6 $10^{-3}$.

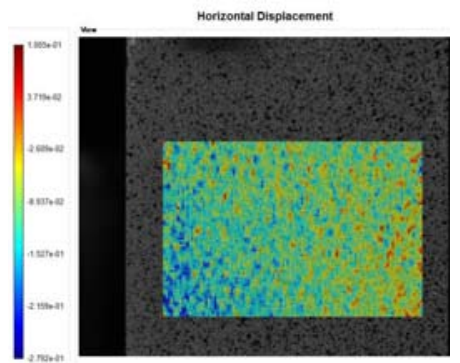

(a) horizontal displacement strin component Ex

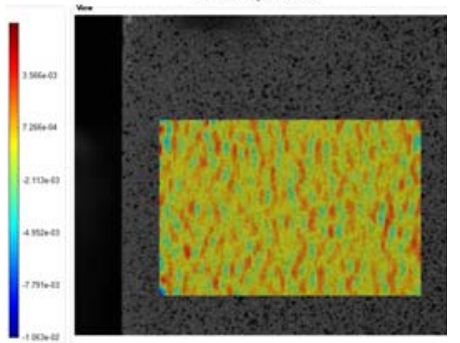

(c) horizontal strain $\varepsilon_{\mathrm{xx}}(\mathrm{w}=5)$ (b) vertical displacement strin componenes Evy

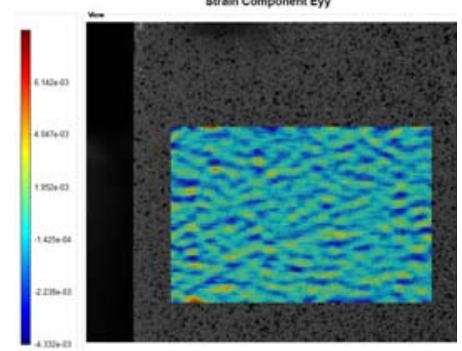

(d) vertical strain $\varepsilon_{\mathrm{yy}}(\mathrm{w}=5)$

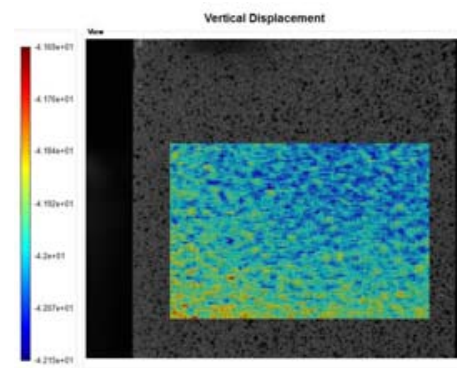

Figure 5 DIC on an experimental imposed rigid body displacement of $1 \mathrm{~mm}$

Using a strain window of 15 lowers the parasitic strain amplitude to about $210^{-3}$ but i) this is in fact still quite large and ii) this type of smoothing may be too extreme to still visualise the strain gradients in a composite. The same applies for increasing the subset size. Doubling the size lowers the amplitude to about $410^{-3}$ for a strain window of 5 , but by increasing the subset, the gradients will be averaged out.

A second unexpected fact is that in Figure 5(a), diagonal areas can be distinguished (top right corner is near red, bottom left is blue) and a similar effect can be seen in Figure 5(b). This effect becomes even more apparent for larger subset sizes. Maybe this is due to a rotation either in-plane or out-of-plane, but it should not be present.

Next, an in-plane rigid body rotation is considered. First, a reference image is taken and then, the specimen is manually rotated over $1^{\circ}$ inside the lower grip. Figure 6 illustrates the results of the correlation and although the displacement fields seem ok, again parasitic strain amplitudes of $510^{-3}$ are present. Changing the subset size did not yield an improvement, but only changed the noise on the images.

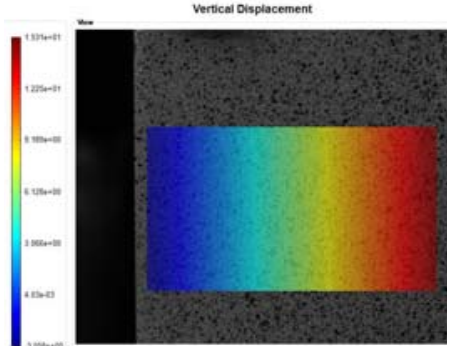

(a) vertical displacement

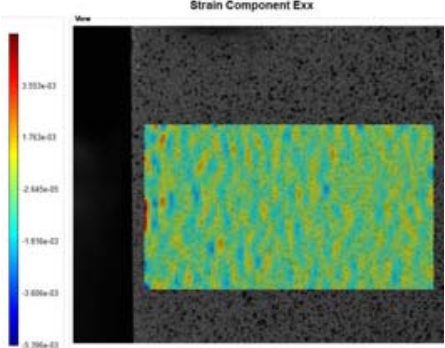

(c) horizontal strain $\varepsilon_{\mathrm{xx}}(\mathrm{w}=5)$

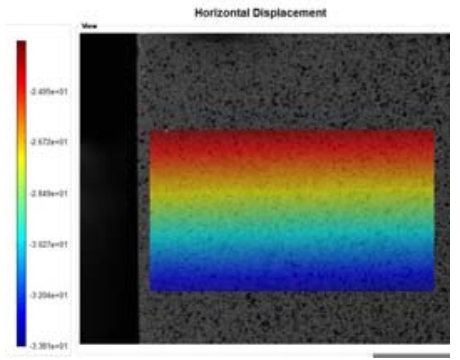

(b) horizontal displacement

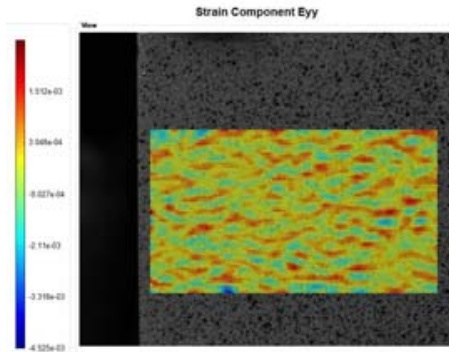

(d) vertical strain $\varepsilon_{\mathrm{yy}}(\mathrm{w}=5)$
Figure 6 DIC on an experimental imposed in-plane rotation

Finally, an out-of-plane rotation was considered. First, a reference picture was taken and then the bottom grip was rotated slightly, after which another photo was taken. Figure 7 illustrates the corresponding results. Again, this rigid body motion induces parasitic strain amplitudes of $810^{-3}$ for $\varepsilon_{x x}$ and $\varepsilon_{y y}$ and $410^{-3}$ for $\varepsilon_{\mathrm{xyy}}$. It should be noted that the diagonal lines are clearly visible in the vertical displacement so probably, a small out-of-plane rotation also happened during the rigid body translation (Fig. 5 (a) \& (b)). On the other hand, these are not the expected displacement fields for a rotation over the vertical axes. Most likely, also a small translation and/or a small in-plane rotation took place, meaning that the depicted displacement fields are the result of such a superposition. 


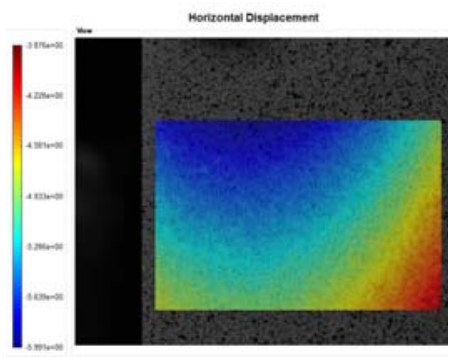

(a) horizontal displacement

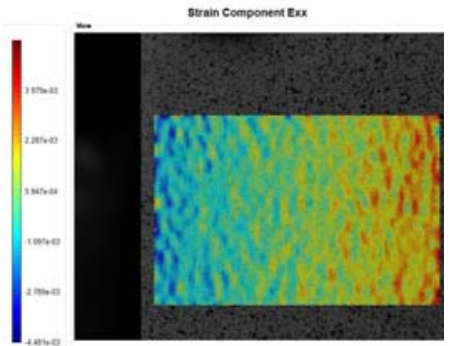

(c) horizontal strain $\varepsilon_{\mathrm{xx}}(\mathrm{w}=5)$

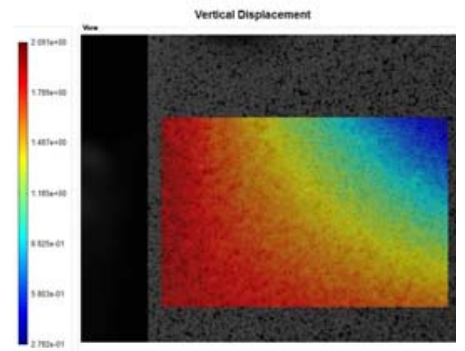

(b) vertical displacement

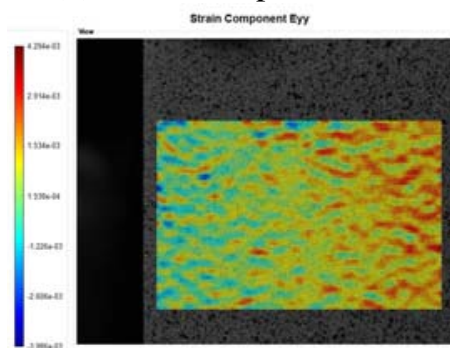

(d) vertical strain $\varepsilon_{\mathrm{yy}}(\mathrm{w}=5)$
Figure 7 DIC on an experimental imposed out-of-plane rotation

\subsection{DIC on numerically induced transformations}

Since the parasitic strains were quite significant for experimentally imposed rigid body transformations and apparently, it is very difficult to experimentally impose a 'pure' rigid body motion, as illustrated by the fact that during the rigid body displacement, a small out-of-plane motion was probably present and vice versa, it seemed necessary to start from the very basic. An image of good correlating speckle pattern, was 'photoshoped' and the following 'virtual' transformations were applied:

i) Horizontal translation of 100 pixels

ii) Vertical translation of 100 pixels

iii) Horizontal strain of $1 \%\left(\varepsilon_{\mathrm{xx}}=0.01\right)$

iv) Vertical strain of $1 \%\left(\varepsilon_{\mathrm{yy}}=0.01\right)$

v) Shear strain $\gamma_{x y}=0.0873$

vi) Rigid in-plane rotation of $1^{\circ}$

vii) Rigid out-of-plane rotation

viii)Combination of in-plane rotation of $1^{\circ}$ and rigid translation of 100 pixels

ix) Combination of in-plane and out-of-plane rotation

To avoid any influence of the speckle pattern on the conclusions, the same experimentally recorded speckle image of the specimen which was considered in the previous paragraph for the rigid body transformations is chosen. By using Photoshop ${ }^{\mathrm{TM}}$, it is assured that all parasitic effects coming from the experimental setup, including optical effects such as lens induced deformation, lighting, vibrations... , are ruled out and only the correlation software will influence the results and the same subset size and stepsize were chosen. Again, the correlation factor varied between 0.99 and 1 , meaning very good correlation is achieved.

Concerning the correlation, the results from vertical or horizontal rigid displacement on one hand and vertical and horizontal strain on the other hand, the results were, of course, similar, so only one of each is discussed her. Figure 8 illustrates the results for a 100 pixel rigid translation and as can be seen (Fig. 8(a)) the value is accurately captured. The strains were calculated using a strain window of 5 for (b) and (c) and in this case, the amplitude of the parasitic strains are in the order of $210^{-4}$ for both strains which is acceptable, although with some imagination, one could see the pattern of a fabric reinforced composite. Increasing the strain window to 15 (Fig. 8(d)) decreases the parasitic strain amplitude to 0.6 $10^{-4}$, but as already mentioned, this may average out the desired strain gradients. Similar remarks apply for the shear strain $\varepsilon_{\mathrm{xy}}$.

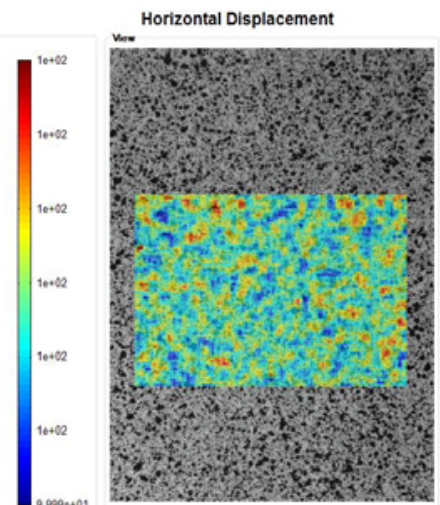

(a) horizontal displacement

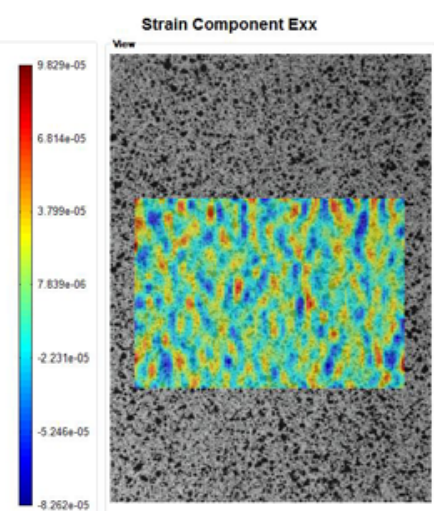

(c) horizontal strain $\varepsilon_{\mathrm{xx}}(\mathrm{w}=5)$

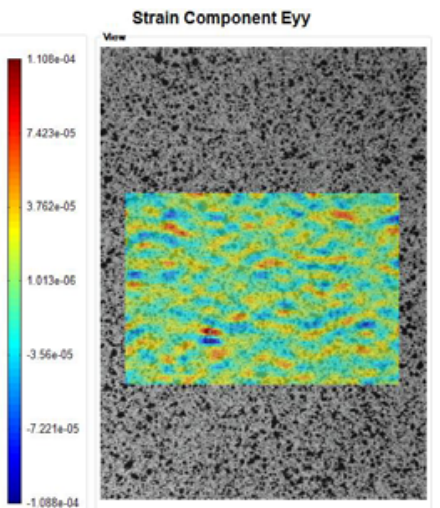

(b) vertical strain $\varepsilon_{\mathrm{yy}}(\mathrm{w}=5)$

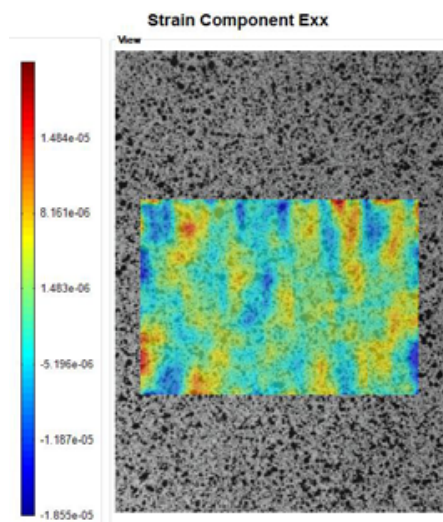

(d) horizontal strain $\varepsilon_{\mathrm{xx}}(\mathrm{w}=15)$
Figure 8 DIC results from 100 pixel horizontal displacement

It should be remarked that the diagonal pattern, which was present in the experimental rigid body translation, is not present here, meaning that this is caused by the experimental setup and as already mentioned, probably due to an extra rigid body transformation.

Figure 9 illustrates the vertical (a) and (parasitic) horizontal (b) strain for the applied 0.01 vertical strain, calculated with a strain window of 5 . The horizontal bands for $\varepsilon_{\mathrm{yy}}$ cannot be missed and the 
difference is in the order of $210^{-3}$, which may be considered fairly large compared to the imposed strain. Recalculating with a strain window of 15 still yields these lines, only thicker, but with the same order of magnitude for the amplitude. The parasitic strain $\varepsilon_{\mathrm{xx}}$ is again in the order of $210^{-4}$ and the same can be said considering the shear strain $\varepsilon_{x y}$.

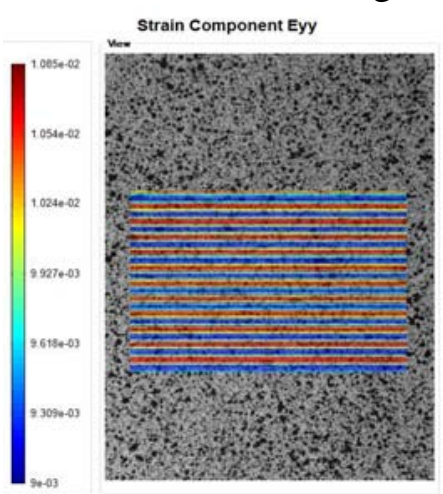

(a) vertical strain $\varepsilon_{y y}$

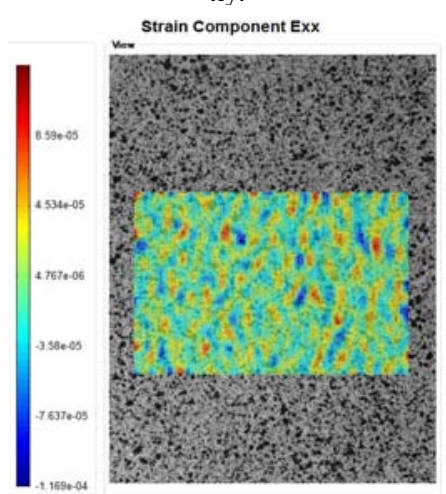

(b) horizontal strain $\varepsilon_{\mathrm{xx}}$
Figure 9 DIC results from 0.01 vertical strain $(\mathrm{w}=5)$

For the induced shear deformation, Figure 10 (a) illustrates the shear strain and as can be seen, the value is predicted accurately, although some noise is present. The parasitic strains (Fig. 10(b)) are a little larger than in the previous cases, a range of $410^{-4}$ is found.

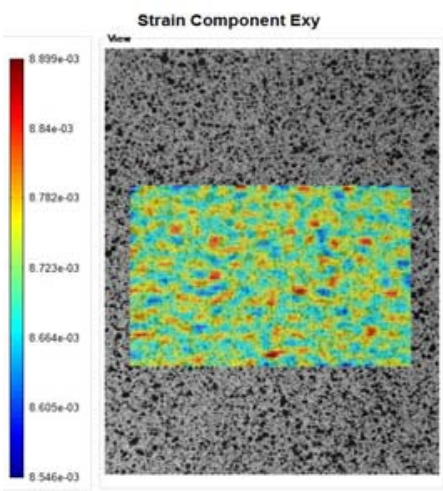

(a) shear strain $\varepsilon_{x y}$

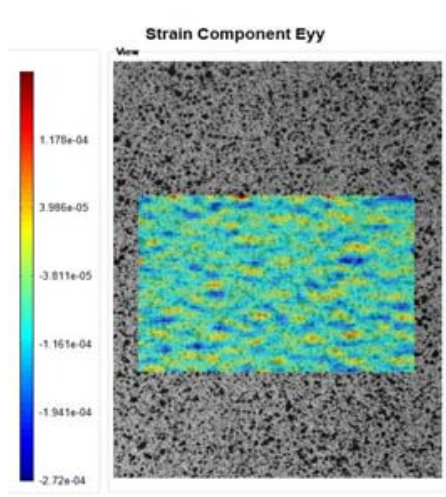

(b) vertical strain $\varepsilon_{y y}$
Figure 10 DIC results from $5^{\circ}$ shear strain $(w=5)$

Next, an in-plane rigid rotation is considered. First, a rotation around the centre of the area of interest was done (Fig. 11(a)), but in reality, it is unlikely that should a rigid rotation occur, the centre would be exactly in the centre of the image, so a second rotation was considered, with the centre outside the image, exactly opposite of the top right corner along the diagonal of the image top right - bottom left (Fig. 11(b)). It should be remarked that both displacement fields are similar to Figure 6 (a) and (b). Figure 9 shows that the amplitude of parasitic strains is about $710^{-4}$. Changing the strain window only smoothed the image and lowering the subset size did not change this value, the latter only increased the noise. The fact that a rigid body rotation causes a larger amplitude lies in the fact that all strain algorithms only compensate for the rigid body rotation of a single subset, not of the entire image.

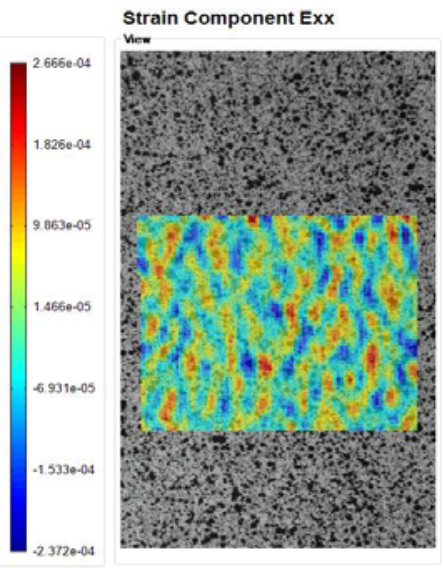

(a) $\varepsilon_{\mathrm{xx}}$ rotation centre photo

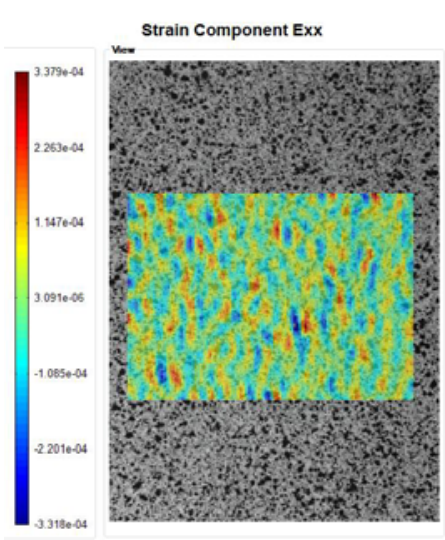

(b) $\varepsilon_{\mathrm{xx}}$ rotation outside photo
Figure 11 DIC results from the rigid body rotation of $1^{\circ}$

For the out-of-plane rotation, the exact rotation angle cannot be set with the used software, this was a manual operation, but since the interest lies in the corresponding deformation trends, rather than the absolute values, this is not really an issue. The results are shown in Figure 12 and as clearly visible, the parasitic strains are significant and more important, with a clear left to right gradient. A top to bottom gradient was visible for the shear strain $\varepsilon_{x y}$. As the exact angle value of the out-of-plane rotation is not known, the amplitude of the parasitic strains has no real value.

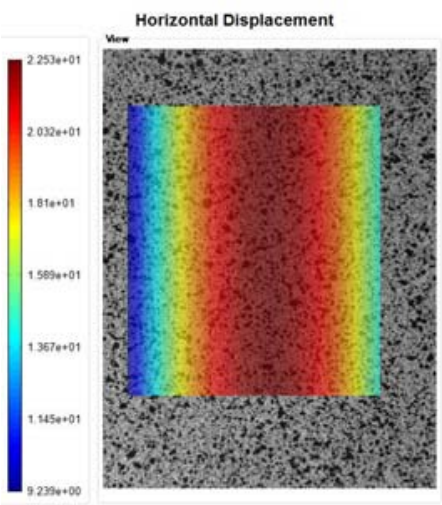

(a) horizontal displacement

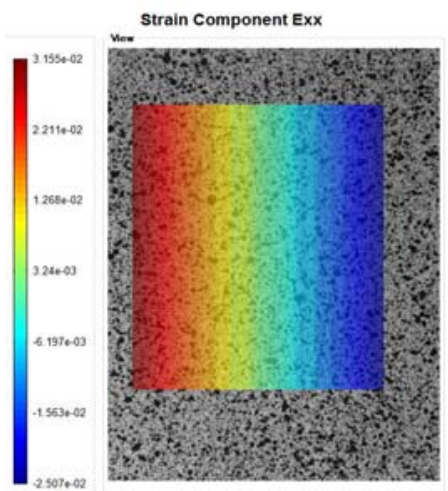

(c) horizontal strain $\varepsilon_{\mathrm{xx}}(\mathrm{w}=5)$

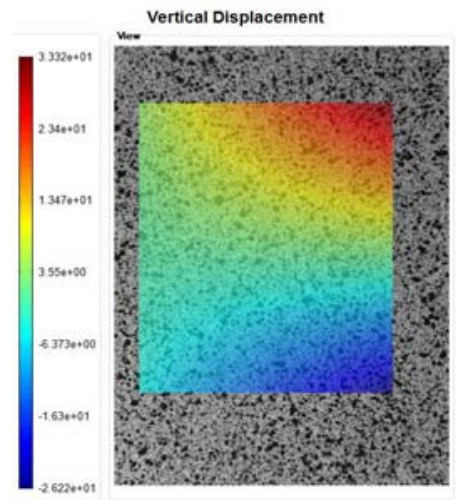

(b) vertical displacement

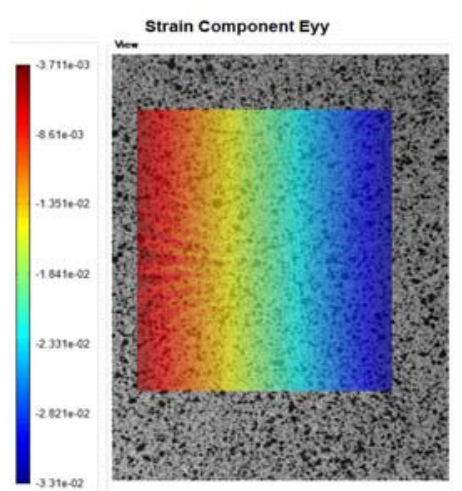

(d) vertical strain $\varepsilon_{\mathrm{yy}}(\mathrm{w}=5)$
Figure 12 DIC from an out-of-plane rotation 
Since the tensile test on the hydraulic Instron (Figure 3) showed displacement fields similar to the ones depicted in Figure 12, despite the presence of the anti-rotation fixture, the latter was subjected to a closer observation. Apparently, there was very little play between the guidance bearings and -rod, allowing for a very small out-of-plane rotation, and as such, the unexpected displacements are clarified.

During all correlations in this paragraph, fields similar to Figure 2 have not yet occurred. As such, combinations of rigid body transformations are now assessed. First, a combination of a rigid body translation and a rigid body rotation with the centre outside the image was considered in order to validate the assumption that the displacement fields in Figure 7 were the result of such a transformation, but this was not the case. Furthermore the amplitude of parasitic strains was equal to the rigid body rotation, meaning that superposing rigid body motions does not mean adding the corresponding parasitic strains.

Finally, a superposition of an in-plane rotation of $1^{\circ}$ and an out-of-plane rotation (same as before) was considered. The centre of rotation for the in-plane rotation was again outside the image, similar to the previous results in Figure 11, the out-of-plane was around the central vertical axis. Figure 13 illustrates both the displacements and the corresponding strains and the correspondence in fields with Figure 7 (and to an extent, Figure 2) cannot be missed.

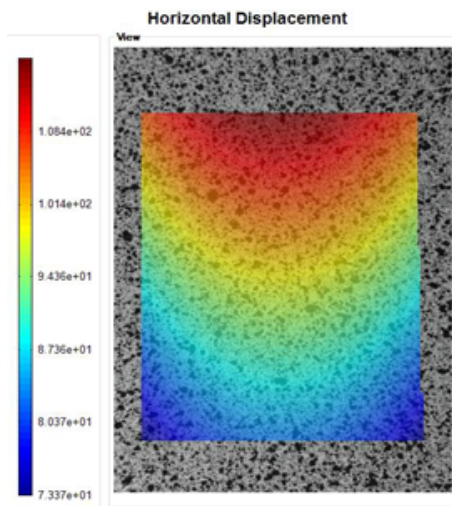

(a) horizontal displacement

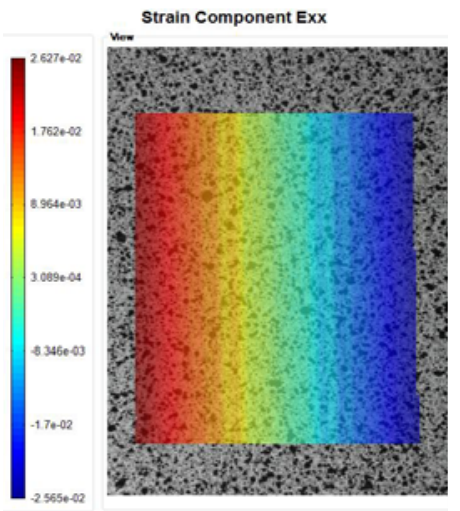

(c) horizontal strain $\varepsilon_{\mathrm{xx}}(\mathrm{w}=5)$

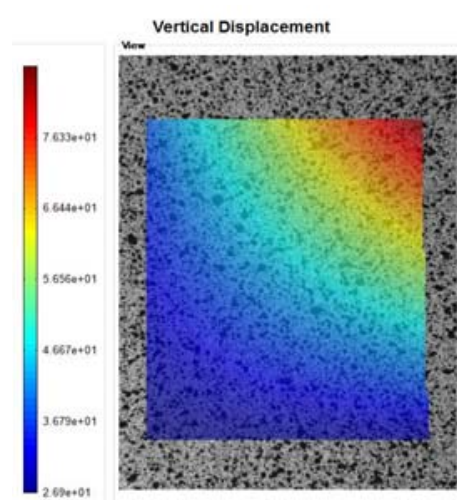

(b) vertical displacement

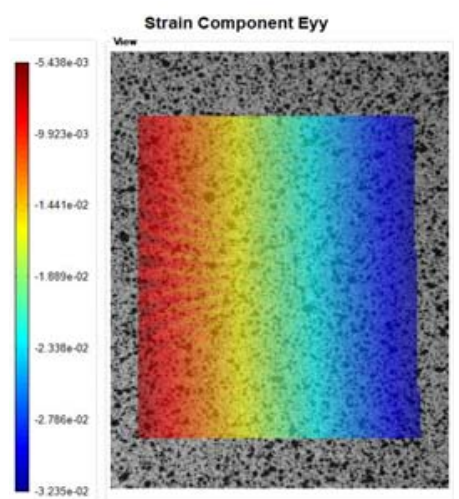

(d) vertical strain $\varepsilon_{\mathrm{yy}}(\mathrm{w}=5)$
Figure 13 DIC results from an in- and out-of-plane rotation.
As such, it may be concluded that parasitic strain levels caused purely by the software algorithms for displacement and strain are fairly small, order $10^{-4}$ for rigid body translation and in-plane rotation.

\section{CONCLUSIONS}

Several experimental and numerical tests were done to assess the accuracy of digital image correlation technique for determining small strains with high gradients. It may be concluded that for pure in-plane rigid body transformations, the error is in the order of $10^{-4}$ and thus negligible, despite the fact that the DIC algorithm only corrects rotation of the subset, rather than rotation of the entire image. Larger parasitic strains should be corrected by playing with subset sizes rather than the strain window, since the latter averages out the gradients which needed to be visualised in the first place. Superposing different in-plane rigid body transformations does not imply superposing the correlation induced errors.

Out-of-plane rotation was also assessed and has a major influence, inducing parasitic strain gradients which cannot be corrected by using other subset sizes, steps or strain windows. Therefore, the experimental setup must be accurately controlled, so that out-of-plane rotation, no matter how small, is inhibited.

\section{REFERENCES}

Lava P. Cooreman S., Coppieters S. De Strycker M. \& Debruyne D. Assesment of measuring errors in DIC using deformation fields generated by plastic FEA. Opt Lasers Eng 2009(47);pp747-753

Van Paepegem W., Shulev A, Roussev I., De Pauw S., Degrieck J. and Sainov V. Study of the deformation characteristics of window security film by digital image correlation techniques. Optics and Lasers in Engineering 2009, 47(3-4) pp 390-397

Willems A., Lomov S.V.,Verpoest A. and Vandepitte D. Drape-ability characterization of textile composite reinforcements using digital image correlation. Optics and Lasers in Engineering 2009, 47 (3-4) pp 343-351 